\title{
DIAGNÓSTICO SOCIO AMBIENTAL DE LA "FINCA LA VIEJA", (MUNICIPIO DE CIMITARRA-SANTANDER) COMO ESTRATEGIA PARA DETERMINAR SU POTENCIAL AMBIENTAL EN LA RECUPERACIÓN DEL ECOSISTEMA
}

\author{
SOCIO-ENVIRONMENTAL DIAGNOSIS OF THE "FINCA LA VIEJA", \\ (MUNICIPALITY OF CIMITARRA-SANTANDER) AS A STRATEGY TO \\ DETERMINE ITS ENVIRONMENTAL POTENTIAL IN THE RECOVERY OF THE \\ ECOSYSTEM
}

\author{
Yenni Alejandra Varón Román \\ yvaronr@unadvirtual.edu.co \\ Alba Lucia Quitian Rojas \\ alquitianr@unadvirtual.edu.co
}

\section{RESUMEN}

La fragmentación de bosques y la degradación de hábitats asociadas son consideradas como las principales causas de la pérdida de biodiversidad en Colombia, es ese sentido que urgen acciones que mitiguen y restablezcan ecosistemas afectados, para ello, una de las estrategias más importantes es la recuperación y restauración de ecosistemas. Sin embargo, ante estas estrategias surgen también una serie de implicaciones relacionadas con la ausencia de información o su falta de accesibilidad, tal como lo son datos actualizados de la flora y fauna amenazada, información satelital de las zonas afectadas, entre otras. En consideración, el presente documento tuvo como objetivo generar un diagnóstico socioambiental a partir de la caracterización del relicto boscoso asociado a la finca "La Vieja" y demás fincas asociadas, con el fin de determinar su potencial ambiental en la restauración del ecosistema local y el mantenimiento de los servicios que ecosistémicos. En ese contexto, el presente diagnóstico se desarrolló considerando factores biofísicos como la vegetación, la fauna y el componente social. Para dicho fin, se tuvo en cuenta información secundaria de instituciones y entidades regionales, así como de información recolectada a partir de recorridos en campo, entrevistas, diálogos con la comunidad y observaciones directa del estado de los principales sistemas boscosos. Como resultado de esta investigación se estimó que el estado actual de las fincas es óptimo en términos de la diversidad de fauna encontrada y del estado sus principales sistemas boscosos, de la misma manera se detectaron zonas de bosque débilmente conectadas por lo cual se propone un mapa de conectividad biológica.

DOI: https://doi.org/10.22490/26653176.4470 
Palabras clave: Ecosistemas, biodiversidad, conectividad, fragmentación, restauración.

\begin{abstract}
Forest fragmentation and associated habitat degradation are considered as the main causes of biodiversity loss in Colombia, in that sense actions that mitigate and restore affected ecosystems are urgently needed, for this purpose, one of the most important strategies are the recovery and restoration of ecosystems. However, a number of implications also arise in these strategies related to the absence of information or its lack of accessibility, such as upto-date data on threatened flora and fauna, satellite information of the affected areas, among others. In consideration, this document aimed to generate a socio-environmental diagnosis from the characterization of the wooded remnant associated with the "La Vieja" property and other associated farms, in order to determine its environmental potential in the restoration of the local ecosystem and the maintenance of ecosystem services. In this context, this diagnosis was developed considering biophysical factors such as vegetation, wildlife, and social component. For this purpose, we take account information from regional institutions and entities, as well as information collected from fieldwork, interviews, community dialogues and direct observations of the forest condition. As a result of this research, it was estimated that the current state of the farms is optimal in terms of the diversity wildlife found and the state their main forested systems, in the same way, weakly connected forest areas were detected so a map of biological connectivity is proposed.
\end{abstract}

Keywords: Ecosystem, biodiversity, connectivity, fragmentation, and restoration.

\title{
1. INTRODUCCIÓN
}

El Magdalena medio santandereano cuenta con una riqueza natural importante que está representada en su diversidad biológica y sus servicios ecosistémicos (Torres \& Quiñones, 2019). Según este mismo autor, el departamento de Santander cuenta con un $20 \%$ de las especies presentes en el país; entre ellos el aporte más grande esta dado por las aves, mamíferos, peces y pantas maderables.

Sin embargo, los bosques y la riqueza de especies de plantas maderables en Santander han sido afectados por el fenómeno de la deforestación, a causa de la tala ilegal, expansión para la ganadería, e incendios forestales, sus principales consecuencias son: Bosques fragmentados, y la grave afectación sobre los hábitats allí presentes, provocando la extinción de muchas especies a nivel local como regional, además afectando el microclima del bosque (Colorado Zuluaga, Vasquez Muñoz , \& Mazo Zuluaga , 2017)., A raíz de esto surge la necesidad de realizar proyectos para identificar zonas de bosques fragmentados y hacer propuestas de restauración del ecosistema.

El presente proyecto de investigación se orientó en identificar la riqueza de la biodiversidad y los servicios ecosistémicos presentes en la vereda Vuelta Acuña del municipio de Cimitarra Santander, por medio de un "Diagnóstico Socio Ambiental De La "Finca La Vieja", (Municipio 
De Cimitarra-Santander) como estrategia para determinar su potencial ambiental en la recuperación del ecosistema, ya que la finca mencionada se encuentra ubicada en un lugar estratégico en medio de dos zonas protegidas la ciénaga del rio viejo y la ciénaga del perico y cuenta con una extensión de terreno de 850 hectáreas aproximadamente, las cuales quieren ser destinados por su propietario como corredor biológico para preservar especies presentes amenazadas y atraer nuevas especies.

Los objetivos propuestos para dar alcance a este estudio fueron: a. Caracterizar el relicto boscoso presente en la finca La Vieja en términos de sus componentes bióticos y abióticos a partir de información secundaria, b. Determinar la percepción de la población con relación a la generación de procesos restaurativos del ecosistema de la finca La Vieja. c.

Analizar la conectividad del relicto boscoso de la finca de La Vieja con relación a los ecosistemas aledaños.

\section{MATERIALES Y MÉTODOS}

El municipio de Cimitarra está localizado al Sur- Occidente del departamento Santander a una distancia de $200 \mathrm{~km}$ de la capital del departamento (Concejo Municipal de Cimitarra Santander, 2016). Dentro de este municipio se emplaza la finca "La Vieja", en la vereda Vuelta Acuña, dicha finca consta de dos terrenos con una extensión total de 850 hectáreas, sus coordenadas; 6³6'57.7"N, 74¹9'16.4"W.

Este diagnóstico se desarrolló mediante un enfoque mixto, evaluando la cantidad y la calidad de flora y fauna presente en la finca "La Vieja" y la "Coqueta".

Para dar alcance a los objetivos propuestos, se desarrollaron 3 fases, las cuales se ejecutaron por medio de información primaria y secundaria recolectada en una (1) salida de campo, la cual se realizó la primera semana del mes de octubre del año 2020 en donde se dio cumplimiento a la siguiente metodología.

Fase 1: Caracterización biofísica del área de estudio a nivel de paisaje- vegetación: En el desarrollo de esta fase se utilizaron las herramientas Google Earth Engine y QGIS, con las que se realizó un análisis de los cambios en la cobertura vegetal, desde los años 20052018, a partir de estos análisis se generaron mapas para identificar la localización del área de estudio, el uso de los suelos y los cambios producidos en la cobertura vegetal en los años arriba mencionados, esto con el fin de analizar cuáles han sido las cusas de la pérdida de la biodiversidad en la región a partir de un análisis comparativo.

Fase 2: Percepción de la Comunidad: El desarrollo de este componente se generó a partir de la comunicación con los pobladores por medio de la aplicación de entrevistas semiestructuradas, la observación en campo y la información secundaria de instituciones locales y regionales, determinando así el uso de bienes y servicios ecosistémicos que las comunidades perciben en el área de estudio. 


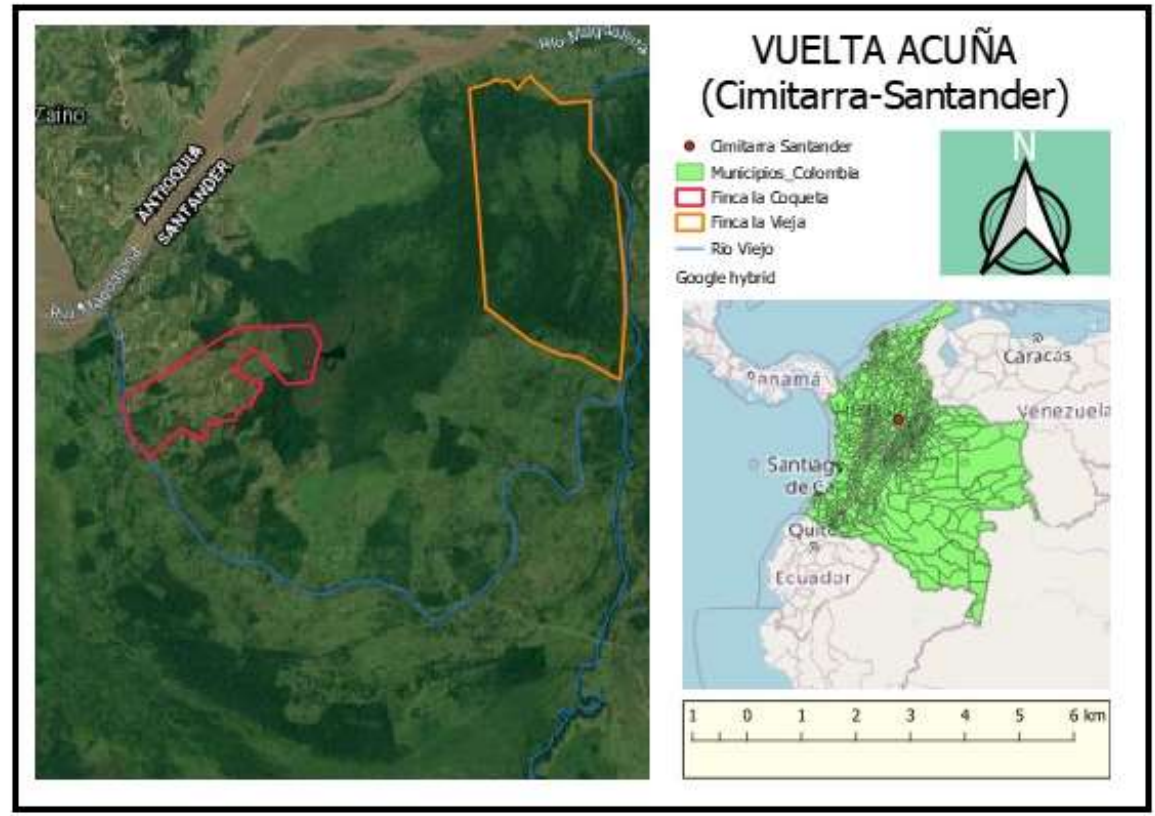

Figura 1. Localización geográfica de la finca. Fuente: Autores, a partir de QGIS.

Se visitaron 10 fincas y se realizaron entrevistas semiestructuradas a ocho personas habitantes de la vereda, se les presento un catálogo ilustrado en donde se seleccionaron los grupos de plantas, aves, peces de agua dulce, mamíferos y reptiles presentes en la región. Al interactuar con la comunidad se generó dialogo, en donde se resaltó principalmente el estado y disponibilidad de especies vegetales, aves y mamíferos, esto de acuerdo con criterios como la importancia que revisten las plantas al considerarse la estructura principal del ecosistema, así mismo, las aves y los mamíferos considerados igualmente importantes debido a que son las especies encargadas de la dispersión de semillas y de la polinización. De esta manera, se constató la información secundaria con la información recolectada en campo y se pudo establecer la importancia que los pobladores de la región le dan al mantenimiento y conservación del ecosistema, considerando así el segundo objetivo de este proyecto.

El catálogo ilustrado se realizó con información de los libros rojos de la Unión Internacional para la Conservación de la Naturaleza (UICN) disponibles para Colombia. Para el caso de las plantas amenazadas se consultó igualmente el catálogo actualizado de Plantas y Líquenes de Colombia de la Universidad Nacional de Colombia, así como también se consultó el informe de la Corporación Autónoma Regional de Santander (CAS) del año 2016.

Fase 3: Caracterización biofísica del área de estudio en campo: Esta caracterización se realizó teniendo en cuenta la flora y fauna presente en la finca "La Vieja" y fincas asociadas, a través de información primaria y segundaria. La disponibilidad de las especies animales y vegetales presentes en la región fueron constatadas en el trabajo en campo, con ayuda de un catálogo ilustrado. De otra parte, también se realizó un muestreo para estimar la diversidad y abundancia de especies vegetales a partir del método Gentry descrito por 
(Chediack, 2009). El muestro se realizó considerando los siguientes criterios; una distancia lineal de 100 metros por 2 metros de ancho y un diámetro a la altura de pecho de $30 \mathrm{~cm}$. El área seleccionada correspondió a un remante de bosque al interior de la finca "La Vieja". Debido a la contingencia por COVID 19, no fue posible realizar sino una visita de campo a la zona por lo que solamente se tiene registro de un transecto.

Con la información recolectada en campo se realizó un análisis estadístico descriptivo con el fin de validar la importancia que la comunidad presenta sobre el medio ambiente y las oportunidades para recuperarlo y mantenerlo. De igual forma se realizó un análisis de la conectividad del relicto boscoso generando un mapa de conectividad del ecosistema de la finca La Vieja con las fincas aledañas y los ecosistemas presentes en esta región, esto con el fin de dar cumplimiento al tercer objetivo.

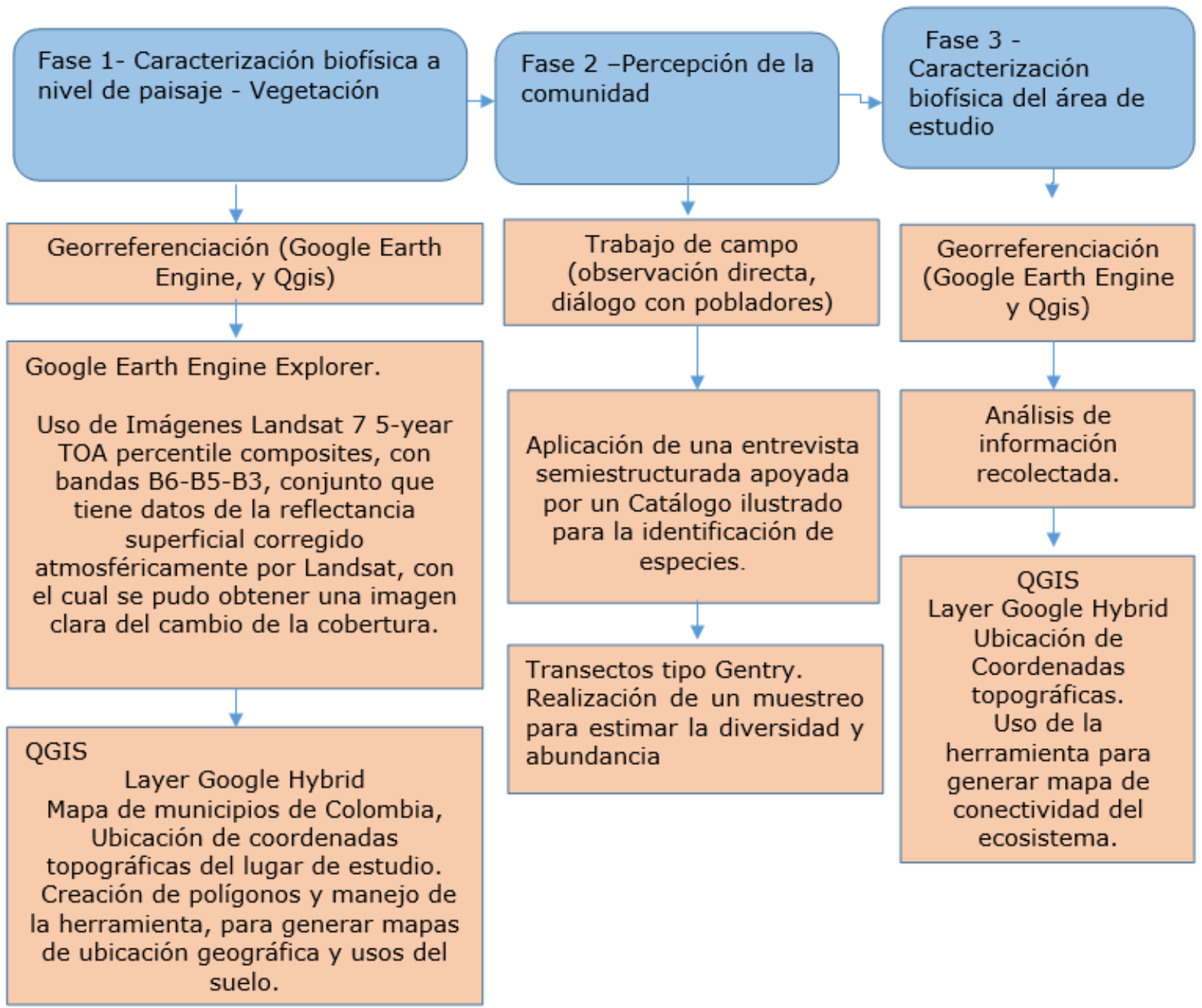

Figura 2. Metodología. Fuente: Autores. Anexo: Entrevista semiestructurada. 


\section{RESULTADOS Y DISCUSIÓN}

\section{Componente biótico}

A partir de la salida a campo, el dialogo con los pobladores, el desarrollo de las entrevistas y la información disponible de las especies amenazadas en la región, se estableció el estado y disponibilidad de los siguientes grupos de animales y especies vegetales.

Tabla 1. Especies de flora y fauna amenazada.

\begin{tabular}{lccc} 
Grupo & $\begin{array}{l}\text { Número de } \\
\text { especies } \\
\text { reportadas en } \\
\text { literatura }\end{array}$ & $\begin{array}{l}\text { Número de } \\
\text { especies } \\
\text { encontradas en la } \\
\text { zona de estudio }\end{array}$ & $\begin{array}{l}\text { Número de } \\
\text { especies } \\
\text { endémicas }\end{array}$ \\
\hline Especies vegetales & 16 & 6 & 6 \\
Aves & 8 & 5 & 4 \\
Mamíferos & 7 & 7 & 4 \\
Peces de agua dulce & 8 & 8 & 6 \\
Reptiles & 5 & 5 & 3 \\
\hline
\end{tabular}

Fuente: Autores.

\section{Especies vegetales}

De acuerdo con los datos recolectados a partir de las entrevistas y los recorridos en campo, de la lista de especies vegetales identificadas como amenazadas en la zona de estudio, las siguientes presentan baja o nula disponibilidad: yumbé (Caryodaphnopsis cogolloi), carreto (Aspidosperma polyneuron), Caoba (Swietenia macrophylla), marfil (Phytelephas macrocarpa), carrá (Huberodendron patinoi) y pino colombiano (Podocarpus oleifolius).

De los anterior se pudo establecer que las bajas densidades de estas poblaciones están relacionadas principalmente con factores como la conversión de bosques a pastizales para la cría de ganado y a la tala de árboles con fines comerciales y a una escala domestica para la construcción de nuevas viviendas y centros de trabajo.

Así mismo, a partir del muestreo de transectos tipo Gentry se encontraron las especies identificadas en la figura 3.

\section{Aves}

De acuerdo con el relato de los pobladores y la observación en campo se pudo establecer que del listado de aves identificadas como vulnerables existen tres que los campesinos declaran no a ver visto en las fincas de esta región, estas son: el cóndor de los andes (Vultur gryphus), el paujil colombiano (Crax Alberti) y el torito capiblanco (Capito hypoleucus). Así mismo, los pobladores expresan que han visto disminuir la presencia en el último tiempo de las siguientes tres aves; el colibrí amazilia ventricastaño (Amazilia castaneiveiventris), el 
cardenalito (Spinus cucullatus) y el pavón moquiamarillo (Crax daubentoni). De las restantes dos aves consideradas amenazadas según los libros rojos para esta región, el águila arpía (Harpía harpyja) y el cucarachero de niceforo (Thryophilus nicefori) cuentan con altas poblaciones aseguran estas comunidades.

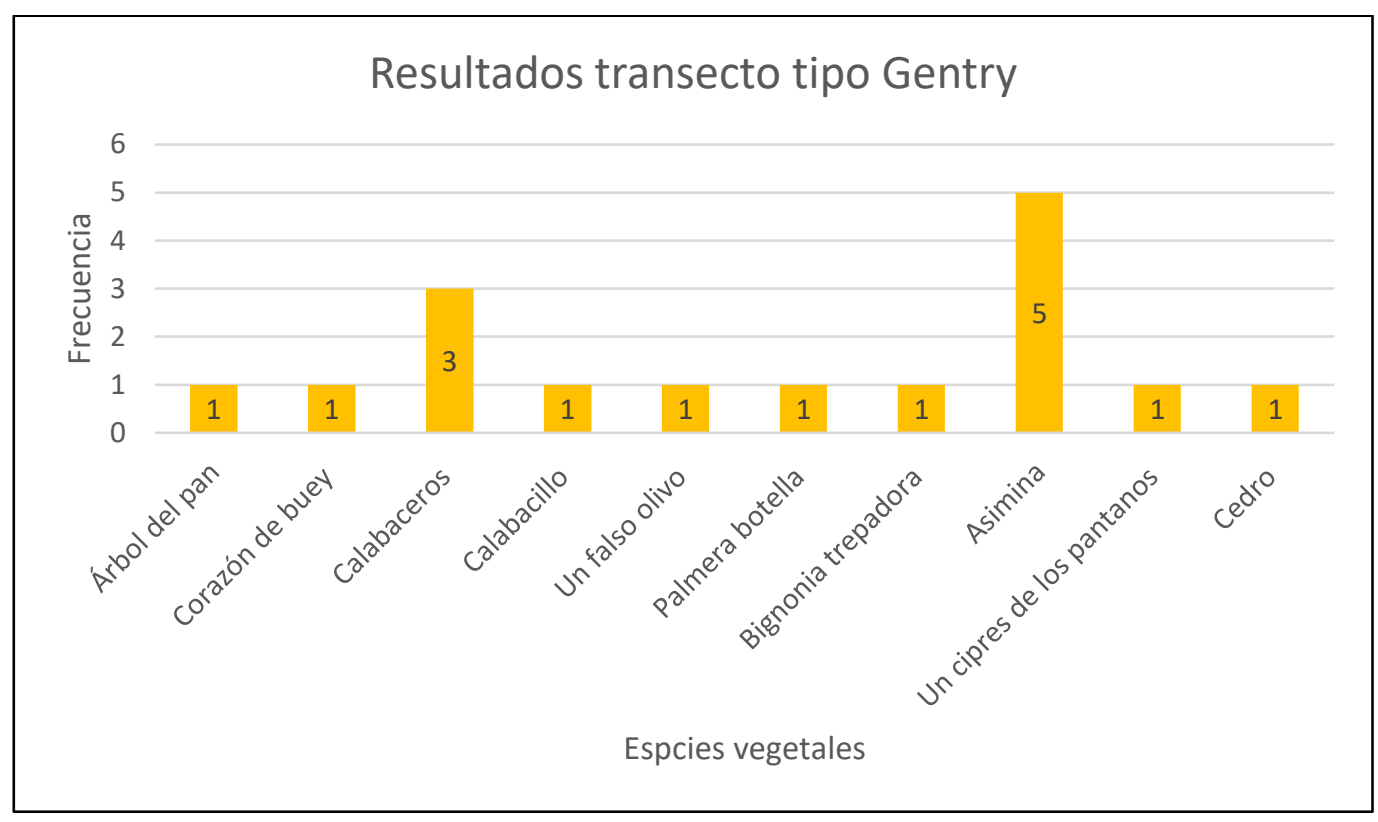

Figura 3. Resultados muestreo tipo Gentry. Fuente: Elaboración propia.

Se puede aludir que la ausencia y las bajas densidades de las especies antes mencionadas corresponden a la pérdida de su habitat por la conversión de bosques a pastizales y por los conflictos que pueden presentarse con la tenencia del ganado.

\section{Mamíferos}

Según los lugareños, la danta (Tapirus terrestres) es la especie más escasa en esta zona, de las ocho personas entrevistadas, seis de ellas indican que la disponibilidad de este animal ha disminuido notablemente, al punto de llegar a ser difícil encontrar algún ejemplar.

De otra parte, la totalidad de los entrevistados manifiestan haber visto o escuchado nombrar sobro el jaguar (Panthera onca), según estas personas, se le ha podido ver a las orillas de los bosques en busca de alimento. Así mismo, el triguillo (Leopardus pardalis), el perro de agua (Speothos venaticus), el mono capuchino (Cebus albifrons) y el oso hormiguero (Myrmecophaga tridactyla) fueron vistos según los entrevistados en el último tiempo, de acuerdo con ellos, estos animales se encuentran representados en la zona con una baja disponibilidad. Finalmente, la marteja (Aotus lemurinus) es de las especies más comunes en la zona, esto de acuerdo con los relatos de los pobladores y en consideración a que durante los recorridos de una jornada de trabajo en campo fue posible observar dos de estos animales. 


\section{Peces de agua dulce}

De los animales considerados como amenazados en esta categoría, se pudo establecer que existen en general una considerable disminución de peces o pescados sobre el rio magdalena y sus afluentes, principalmente del bagre (Pseudoplatystoma magdaleniatum) y el jetudo (Ichthyoelephas longirostris).

Esta disminución consideramos que está relacionada con su consumo masivo, ya que contribuye a completar la dieta de los habitantes, y es así mismo la única fuente de trabajo para otros lugareños, los cuales extienden el comercio de pescado para las veredas aledañas y Puerto Berrio, esto según se pudo establecer a partir del dialogo con pescadores.

\section{Reptiles}

Sobre las especies de reptiles, la mayoría de las personas consultadas aseguran que en la zona existe una buena disponibilidad de las especies identificadas como vulnerables según los libros rojos, con la excepción de la culebra coral (Micrurus sangilensis). Esta especie fue identificada como escasa o poco común. Pudimos establecer que su baja disponibilidad en la zona está directamente relacionada con el peligro físico que representa para los pobladores y sus animales, por lo que su habitat se ve reducida casi exclusivamente a los bosques primarios.

Po otra parte, se resalta que la zona es ampliamente habitada por babillas o también conocida como caimán aguja (Crocodylus acutus); se puede establecer que su amplia presencia en la zona esta favorecida por la alta anegación que presentan los suelos de las fincas analizadas; tal como se pudo constatar, es común encontrar este animal en épocas de invierno a la orilla de los diferentes pantanos y cerca del ganado.

\section{Mapa de conectividad biológica}

A continuación, se propone el mapa de conectividad biológica del ecosistema de las fincas "La Vieja", "La Coqueta" y sistemas aledaños, considerado criterios funcionales de los componentes faunísticos y florísticos identificados en campo.

A partir de los recorridos en campo y del análisis de imágenes satelitales evidenciamos zonas débilmente conectadas al interior de las fincas con el sistema de bosques adyacente a la ciénaga el Perico, por lo que surge la necesidad de generar programas de reforestación sobre las áreas fragmentadas que en la actualidad no presentan conectividad con el sistema boscoso y consecuentemente dificultan el tránsito de especies de animales; de esta manera se proyectó el mapa de conectividad del relicto boscoso (figura 4), donde se identifican las áreas que deben ser consideradas para tal fin.

Se estima entonces que a partir de la restauración de los fragmentos de bosque señalados y voluntad de sus comunidades por la protección del bosque, este ecosistema represente más 
y mejores condiciones para el sostenimiento del hábitat de las diferentes especies y para el mantenimiento de los servicios ecosistémicos. En dicho sentido se resalta la serie de servicios que el ecosistema actual provee a la comunidad, como lo es el servicio de provisión de alimentos, de agua y madera, servicios de regulación como la regulación del clima y del ciclo del agua, el control de la erosión del suelo y la polinización, servicios de soporte como la biodiversidad y servicios culturales como la recreación el turismo y la inspiración espiritual.

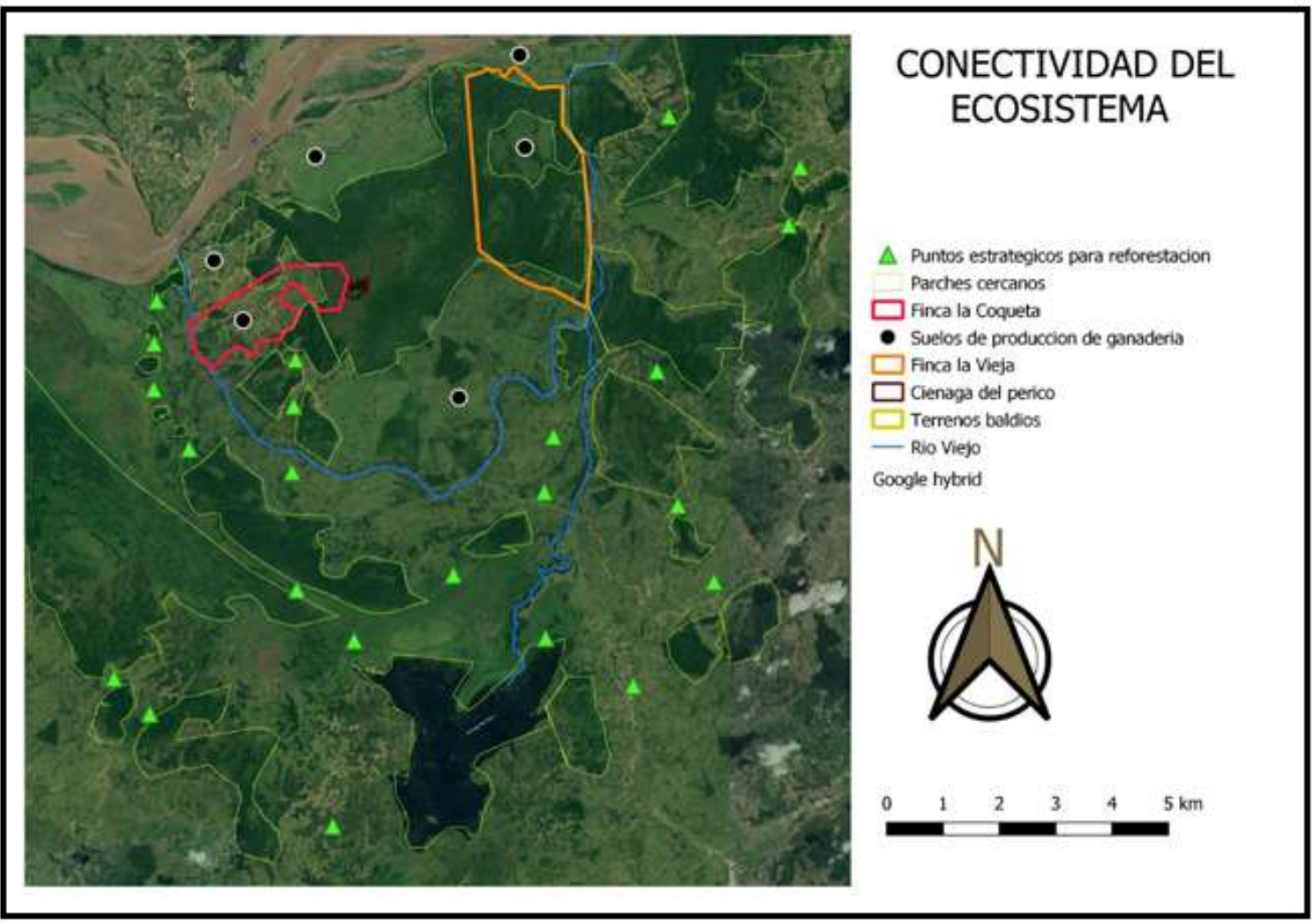

Figura 4. Mapa de conectividad biológica. Fuente: Elaboración propia a partir de QGIS.

\section{Componente socioeconómico}

Durante la fase de campo se visitaron 10 fincas en la vereda Vuelta Acuña y se realizaron un total de ocho entrevistas. A partir de dichas entrevistas se determinó una caracterización socioeconómica descrita a continuación:

\section{Economía}

La cría de ganado con triple propósito (leche, carne y trabajo) constituye la principal actividad económica de la vereda, en relación con la empleabilidad de mano de obra, esta actividad requiere de vaqueros, encargados y administradores. así mismo esta actividad involucra empleados para oficios varios, especialmente la limpieza de potreros. 
Dentro de las razas de ganado identificadas a partir de las entrevistas y de la observación en campo, se encuentran; el ganado nelore, el cebú rojo, el cebú blanco, el gyr, el angus y el ganado blanco orejinegro.

Relacionado a la cría de ganado se identificaron actividades como la tala de árboles, principalmente para la apertura de nuevos potreros para la cría, así como insumo para la construcción de cercas y de nuevas viviendas.

De otra parte y a una escala menor se identificó que la pesca con fines comerciales y para el abastecimiento familiar constituye otra fuente de trabajo para algunas comunidades rivereñas.

Otra de las actividades comúnmente desarrolladas en esta región es la caza animal, principalmente de chigüiros (Hydrochoerus hydrochaeris) y del tapir (Tapirus terrestris). Dicha actividad ha venido en descenso de acuerdo con el relato de los pobladores debido a su escaza disponibilidad y a su prohibición. Se trata de acuerdos comunes entre los diferentes propietarios que prohíben a sus empleados y demás habitantes la caza de cualquier animal en estos terrenos.

Como complemento del abastecimiento de alimentos, los habitantes perciben otros beneficios del suelo relacionado con árboles frutales, entre ellos se cuenta con, toronjas, limones, mandarina, mangos, guayabas y papayas. Así mismo se identificaron algunos tubérculos, entre ellos, yuca y plátano. De otra parte, la cría de aves de corral para el consumo como gallinas y pavos domésticos contribuye a completar la dieta de los pobladores. Cabe aclarar que tanto la cría de animales como la plantación de los diferentes arboles se realiza a una escala domestica para suplir únicamente las necesidades del hogar.

\section{Educación}

La vereda vuelta acuña cuenta en la actualidad con una única escuela rural, se trata de la Institución educativa San José. Este plantel ofrece los niveles de preescolar, básica y primaria.

A partir de los recorridos en campo también se pudo determinar la existencia de otra escuela rural en predios de la finca la "Coqueta", propiedad del mismo dueño de la finca "La vieja".

\section{Salud}

En la zona de estudio no se encontraron centros de atención en salud, de esta manera los habitantes que requieran atención medica deber asistir al pueblo más cercano que es Puerto Berrio, el cual se ubica a 1 hora aproximadamente en canoa.

\section{Vivienda}

En la vereda Vuelta acuña predomina las típicas viviendas construidas con paredes en madera mayormente y techo de teja de zinc. Algunas pocas viviendas también están construidas en 
cemento y otras con cerramientos en malla, lo que evita el paso de zancudos. Cada una de estas viviendas están apartadas unas de las otras a lo largo de la ronda del cauce del rio Viejo, así mismo se identificó que son habitadas principalmente por los administradores y trabajadores.

\section{Servicios públicos y saneamiento básico}

\section{Energía eléctrica}

En general todas las viviendas cuentan con este servicio, de manera que esto les permite la tenencia de electrodomésticos y cercas eléctricas para el cerramiento de potreros.

\section{Gas domiciliario}

Las poblaciones asentadas en la zona de interés no cuentan con dicho servicio. Para la preparación o cocción de alimentos utilizan leña y carbón mayormente, en una menor proporción se utiliza cilindros de gas propano.

\section{Agua potable}

El suministro de agua potable es el mayor reto para estas poblaciones, en la actualidad las fuentes de consumo provienen de las aguas lluvias, perforados y en una menor proporción proviene de los cuerpos de agua cercanos. El agua recolectada a partir de todas estas fuentes es almacenada individualmente en tanques elevados ubicadas en las diferentes fincas de la vereda, así mismo, se identificó que el agua sustraída especialmente de perforados debe pasar por un proceso primaria de tratamiento a base de hipoclorito de sodio, esto con el objetivo de remover el exceso de hierro presente. Cabe destacar que este único sistema está ubicado en la finca principal "La Vieja" y sus usos están orientados a satisfacer las necesidades de dicha finca, así como los requerimientos de la actividad ganadera.

\section{Gestión de excretas}

En general en todas las viviendas en las que se aplicaron entrevistas cuentan con un sistema para el manejo de excretas, el cual consiste en una caseta en cemento dotada de un sanitario y fosa séptica.

\section{Manejo de residuos solidos}

La disposición final de los residuos sólidos se maneja de manera independiente en cada finca. En su mayoría estas comunidades establecen un área dentro de sus terrenos para recolectar los residuos que luego incineran. En algunas otras viviendas se opta por la separación de los residuos orgánicos los cuales utilizan posteriormente como abono para el jardín o para árboles frutales. 


\section{CONCLUSIONES}

A partir de los hallazgos encontrados es posible establecer que a pesar del grado de alteración que se ha presentado en la zona de estudio en los últimos años, las condiciones actuales de los ecosistemas presentes en la finca La Vieja y fincas asociadas es óptimo en cuanto a la disponibilidad y calidad de sus recursos. Se pudo establecer que existen aún una buena disponibilidad de bosques primarios, los cuales constituyen el hábitat de especies amenazadas como el jaguar (Panthera onca), el tigrillo (Leopardus pardalis) y la marteja (Aotus lemurinus). Podemos concluir que la presencia de estas especies en la zona indica que existen buenas condiciones de hábitat para una importante población dentro del ecosistema, esto considerando los altos requerimientos que estas especies demandan para su subsistencia.

Así mismo se resalta que la baja densidad poblacional sobre esta vereda, junto con la prohibición por parte los propietarios sobre la caza de cualquier especie, ha mejorado el tránsito de los animales, ya que estos tienen más libertad de movilizarse sin riesgo de ser amenazados. Este tipo de medidas ha contribuido de manera importante al libre desplazamiento y a la confianza que pueden presentar algunos animales. No obstante resulta conveniente desarrollar programas de educación ambiental que permitan mejorar la convivencia con especies depredadoras como el jaguar (Panthera onca), ya que estos representan el riesgo más grande para las comunidades y sus actividades económicas. En este sentido, es importante generar mayor conocimiento en los habitantes locales sobre el cuidado e importancia que revisten estas especies dentro de los ecosistemas.

Por otra parte, destacamos la buena gestión de los potreros dedicados a la cría de ganado, como se logró constatar, estrategias como el silvopastoreo garantizan condiciones para la supervivencia de otras especies. Los sistemas silvopastoriles están ideados de manera que se evite la sobrecarga de los potreros al tiempo que contribuyen con la conectividad biológica de especies; en este caso, animales como las babillas (Crocodylus acutus), osos hormigueros (Myrmecophaga tridactyla), ardillas (Sciurus vulgaris), garzas (Ardeidae), cuervos (Corvus corax), aves pequeñas entre otros. En ese sentido se recomienda a los pobladores considerar la introducción de razas de ganado nativas, debido a la mejor capacidad que presentan de protección del medio ambiente al adaptarse a zonas degradadas.

Finalmente merece especial atención la forma como en la actualidad se realiza la disposición de los residuos sólidos en cada finca, tal y como se evidenció, estos son dispuestos a cielo abierto para luego ser incinerados o enterrados en inmediaciones de las viviendas, ocasionando de esta manera un fuerte impacto en el paisaje y riesgos para la salud de las personas y de la fauna silvestre. En ese sentido es imperativo dar un adecuado tratamiento a los residuos sólidos generados al interior de cada finca, se propone el establecimiento de una adecuada separación en la fuente y un posterior uso de los residuos orgánicos para ser destinados como abono para las plantas o completar la dieta de los animales con estos restos orgánicos. En tanto al manejo de residuos inorgánicos se recomienda en primer lugar, 
reemplazar al máximo por envases biodegradables y en último lugar generar un punto de acopio de estos para luego poder ser entregados en un centro de reciclaje de Puerto Berrio.

\section{CONTRIBUCIÓN DE LA AUTORÍA}

Primer autor: administrador del proyecto, investigación, escritura, metodología, análisis de los datos, logística, revisión y edición. Segundo autor: escritura, análisis de datos, georreferenciación.

\section{AGRADECIMIENTOS}

Agradecemos a la comunidad de la vereda "Vuelta Acuña" por su cooperación en el diligenciamiento de las entrevistas, por su disposición en la transmitirnos de su conocimiento sobre el territorio. Así mismo agradecemos al Sr Carlos Crison, propietario de las fincas de estudio, por su contribución financiera, logística e intelectual que hicieron posible este proyecto. Finalmente agradecemos a la directora del proyecto, Denisse Viviana Cortes por sus conocimientos, orientación y asistencia permanente sobre este proyecto.

\section{REFERENCIAS}

Colorado Zuluaga, G., Vasquez Muñoz, J., \& Mazo Zuluaga , I. (2017). Modelo de conectividad ecológica de fragmentos de bosque andino en santa elena (medellín, colombia). Scielo. doi: http://dx.doi.org/10.15446/abc.v22n3.63013

Instituto de Hidrología, Metereología y Estudios Ambientales. (2020, Octubre 1). Seguimiento y monitoreo de la deforestación en Colombia Primer trimestre de 2020. Retrieved from http://ideam.gov.co/documents/10182/110979891/comdeforestacionboletin22/b592 b0bf-957b-44db-87f5-ae6e489ff971

Montero, I., López, R., Cárdenas, D., \& Salinas, N. (2007). Libro rojo de plantas de Colombia. Volumen 4. Especies maderables amenazadas, Primera parte. Serie Libros Rojos de Especies Amenazadas de Colombia. In Instituto Amazónico de Investigaciones Científicas SINCHI - Ministerio de Ambiente, Vivienda y Desarrollo Territorial. https://www.sinchi.org.co/files/publicaciones/publicaciones/pdf/LR MADERABLES.pd $\underline{f}$

Torres, M., \& Quiñones, C. (2019). Santander Bio:Resultados, Retos y Oportunidades. Instituto de investigacion de Recursos Biologicos Alexander Von Humboldt, BogotaColombia. Recuperado el 28 de febrero de 2020, Disponible en: http://repository.humboldt.org.co/handle/20.500.11761/35339

Universidad Nacional de Colombia. (S/F). Catálogo de plantas y líquenes de Colombia. Obtenido de http://catalogoplantasdecolombia.unal.edu.co/es/

\section{ANEXOS}

Anexo - Entrevista semiestructurada 